\title{
Erratum for Vol. 57, No. 1, page 49: Adaptive Nonlinear Control for a Reusable Space Vehicle's Longitudinal Dynamics under Model Uncertainties
}

\author{
By Kwangsoo KIM ${ }^{1)}$ and Hyochoong BANG ${ }^{2)}$
}

${ }^{1)}$ NARO System Integration Department, Korea Aerospace Research Institute, Daejeon, Republic of Korea

${ }^{2)}$ Department of Aerospace Engineering, Korea Advanced Institute of Science and Technology, Daejeon, Republic of Korea

An error has been found in the first author's affiliation.
Wrong:
Kwangsoo $\mathrm{KIM}^{1)}$
Right:
Kwangsoo $\mathrm{KIM}^{1), 2)}$ 